\title{
Clinical and Computed Tomography (CT) Characteristics of Pulmonary Nodules Caused by Cryptococcal Infection
}

\author{
Fang Chen ${ }^{1,2, *}$ \\ Yuan-Bing Liu ${ }^{1,3, *}$ \\ Bin-Jie Fu (D) \\ Fa-Jin Lv' \\ Zhi-Gang Chu (1D)
}

'Department of Radiology, The First Affiliated Hospital of Chongqing Medical University, Chongqing, People's Republic of China; ${ }^{2}$ Department of Radiology, Youyang Hospital, A Branch of The First Affiliated Hospital of Chongqing Medical University, Chongqing, People's Republic of China; ${ }^{3}$ Department of Radiology, Qijiang People's Hospital, Chongqing, People's Republic of China

*These authors contributed equally to this work
Correspondence: Zhi-Gang Chu Department of Radiology, The First Affiliated Hospital of Chongqing Medical University, I\# Youyi Road, Yuanjiagang,

Yuzhong District, Chongqing, 400016,

People's Republic of China

Tel +8618723032809

Fax +8623688II487

Email chuzg0815@I63.com
Purpose: To investigate the clinical and computed tomography (CT) characteristics of pulmonary nodules caused by cryptococcal infection and gain a greater insight and understanding that will reduce misdiagnosis.

Materials and Methods: A total of 47 patients with confirmed pulmonary cryptococcosis (PC) manifested as nodules were retrospectively enrolled from January 2013 to December 2020. The clinical and CT data of patients with single and multiple nodules were analyzed and compared with emphasis on exploring the characteristics of the solitary ones.

Results: Among the 47 patients, single and multiple nodules were detected in $25(53.2 \%)$ and $22(46.8 \%)$ patients, respectively, with similar clinical characteristics. The diameter of solitary nodules was smaller than that of the largest ones of the multiple nodules $(P=0.000)$. Compared with multiple nodules, less solitary nodules were located in the subpleural zone $(P=0.031)$ and had a cavity or vacuole $(P=0.049)$. Regarding the solitary nodules, 22 $(88.0 \%)$ and $3(12.0 \%)$ were solid and subsolid, respectively. Eighteen $(81.8 \%)$ solid solitary nodules exhibited either one of air bronchogram or vacuole, satellite lesions, and halo sign. Twenty-three $(92.0 \%)$ of 25 patients with solitary nodules had follow-up CT data, and most of them had no changes $(12,52.1 \%)$ or increased in size and/or density $(8,34.8 \%)$, but only a few $(3,13.0 \%)$ decreased with or without anti-inflammatory therapy.

Conclusion: The clinical characteristics of patients with solitary and multiple nodules caused by PC are similar. The solitary nodules are smaller, more scattered, but less necrotic than multiple ones. For solitary nodules with air bronchogram or vacuole, satellite lesions, or halo sign, PC should be considered as one of the differential diagnosis.

Keywords: tomography, x-ray computed, pulmonary nodules, cryptococcosis

\section{Introduction}

Pulmonary cryptococcosis (PC) is an illness caused by $C$. neoformans infection, which occurs sporadically worldwide. Most cryptococcal infections occur in immune-compromised patients, ${ }^{1}$ but they can also develop in immune-competent individuals. $^{2,3}$ In recent years, with the application of glucocorticoids, immunesuppressants, and antitumor drugs, the prevalence of cryptococcal infection has increased. Pulmonary lesions in patients with cryptococcal infections may manifest as single nodules, multiple clustered nodules, multiple scattered nodules, and masslike or pneumonic opacity. ${ }^{4}$ Clinically, compared with other pulmonary infections, the cryptococcal infection, especially in immunocompetent patients, is rather indolent and usually appears as a localized nodule or a mass. ${ }^{5,6}$ 
Currently, our understanding regarding PC is mostly limited to its typical clinical presentations and radiological manifestations. However, the clinical and imaging features of PC are nonspecific. In patients with PC, solitary and multiple nodules are the two main manifestations of pulmonary lesions. However, the differences in clinical and imaging characteristics between these two patterns are still unknown. Additionally, in contrast to the multiple nodular pattern, the single nodular pattern may mimic malignant tumors, chronic inflammation, or a metastatic lesion in patients with malignancy. ${ }^{7,8}$ This renders the imaging diagnosis of this disease as significantly challenging, as it may lead to misdiagnosis. Thus, it is significant to reveal the clinical and imaging features of solitary nodules caused by PC and gain a greater insight and understanding that will in turn reduce misdiagnosis.

Previous studies on PC have mainly investigated the computed tomography (CT) features of different patterns of lesions, and the single and multiple nodules are usually described together. ${ }^{9,10}$ In contrast, the clinical and imaging characteristics of solitary nodules have not been fully revealed. Thus, our knowledge on diagnosing single nodular pattern PC is limited. In this study, we only included the pathologically confirmed single and multiple nodular patterns of PC. Furthermore, their clinical and CT data were analyzed and compared with emphasis on investigating the characteristics of the single nodular pattern, which may be helpful for improving the diagnosis.

\section{Materials and Methods}

\section{Patient Selection}

The study sample was selected between January 2013 and December 2020 from the First Affiliated Hospital of Chongqing Medical University in China based on the following inclusion criteria: (1) PC was histologically confirmed by either biopsy or surgical resection; (2) the pulmonary lesions were only solitary or multiple nodules $(\leq 3 \mathrm{~cm})$; and (3) complete clinical and CT data were available. Patients were excluded if their $\mathrm{CT}$ images were of poor quality or if their multiple nodules were consistent with a miliary pattern. Finally, a total of 47 patients were included in this study, of which 37 cases had undergone operation (thoracoscopic surgery), whereas the remaining 10 cases had been confirmed by biopsy (CTguided percutaneous needle biopsy). The $C$. neoformans were determined by histological examination with combination of morphological findings (hematoxylin-eosin staining) and periodic Acid-Schiff with diastase (PAS-D) or grocott methods.

\section{CT Protocol}

All patients were examined with a 64-slice spiral CT scanner (SOMATOM Definition Flash, Siemens, Germany) with the following settings: tube voltage, $140 \mathrm{kVp}$; tube current calculated according to individuals' weight, height, and body mass index; rotation time, $0.5 \mathrm{~s}$; pitch, 1.0 ; collimation, $0.6 \mathrm{~mm}$; and slice thickness and interval for axial images, 5 and $5 \mathrm{~mm}$, respectively. Thin-section images were reconstructed: $1.0 \mathrm{~mm}$ slice thickness with $0.8 \mathrm{~mm}$ interval. Upon CT examination, patients were placed in the supine position with both hands near their heads. Image acquisition was performed from the level of the thoracic inlet to inferior to the costophrenic angle. All patients underwent plain $\mathrm{CT}$ scanning, and 21 patients underwent contrast-enhanced CT scan with a total of $80-100 \mathrm{~mL}$ of nonionic iodinated contrast material (Iopamidol, $320 \mathrm{mg}$ / $\mathrm{mL}$; Shanghai Bracco Sine Pharmaceutical Co., Ltd., China) at an injection rate of $3.0 \mathrm{~mL} / \mathrm{s}$, followed by $50 \mathrm{~mL}$ of saline solution via a power injector. Images were obtained with mediastinal (width, 350-400 HU; level, 20-40 HU) and lung (width, 1200-1600 HU; level, -500 to $-700 \mathrm{HU}$ ) window settings.

\section{Image Analysis}

Two independent and experienced radiologists, who were blinded to the data, analyzed all studies. Discordant opinions were resolved by consensus. The characteristics of lesions on both plain and enhanced CT scans were combined. Pulmonary nodules were classified into two main patterns: single nodular and multiple nodular. In patients with multiple nodules, the clustered nodular pattern was defined when the lesions consisted of multiple nodules confined to one or two segments, whereas the scattered nodular pattern was defined when lesions were scattered throughout one or multiple lobes. We evaluated the following parameters not only in solitary nodules but also in the largest ones of the multiple nodules on CT images: nodule size (the mean of the longest diameter and the perpendicular diameter on axial CT images), location (upper, lower, or middle lobe), shape (round, oval, or irregular), density (solid or subsolid), CT value on plain CT scan, degree of enhancement (peak CT value on contrast-enhanced $\mathrm{CT}$ scan - CT value on plain CT scan), air bronchogram (yes/no), vacuole sign (yes/no), margin (smooth or coarse), boundary (well-defined or ill-defined), spiculation (yes/ 
no), halo sign (yes/no), concomitant satellite lesions (yes/ no), and pleural indentation (yes/no). Subsolid nodules included pure ground-glass nodules (pGGNs) and mixed ground-glass nodules (mGGNs). Pleural effusion and lymphadenopathy (when the short-axis diameter was greater than $10 \mathrm{~mm}$ ) were also recorded.

\section{Statistical Analysis}

The patients' clinical data and CT features of nodules were statistically analyzed using the SPSS software package (version 22.0, IBM, NY, USA). Continuous variables were expressed as mean \pm standard deviation, and categorical variables were expressed as number and percentage. To compare differences among different patterns of nodules, the Mann-Whitney $U$-test was used for patients' age and nodules size, and the Pearson chi-square test was used for gender, symptoms, basic diseases, and frequencies of different CT features. A P-value of $<0.05$ was considered as statistically significant difference.

\section{Results}

\section{Clinical Findings}

Among the 47 patients with PC, there were 28 men and 19 women, with a mean age of $47.0 \pm 12.8$ years (range, 23 74 years). Single nodule and multiple nodular patterns were detected in $25(53.2 \%)$ and 22 (46.8\%) patients, respectively. Table 1 summarizes the clinical characteristics of patients with solitary and multiple nodules. In both groups, patients' gender, mean age, symptoms, and concomitant basic diseases were similar (each $P>0.05$ ). Two patients with a single nodule (8.0\%) and six (27.3\%) patients with multiple nodules presented with symptoms of headache and were confirmed to suffer from cryptococcal meningitis. Initial CT diagnoses of these nodules included pulmonary tuberculosis $(8,17.0 \%)$, primary tumors $(11,23.4 \%)$, inflammatory granulomas (15, $31.9 \%)$, metastases $(2,4.3 \%)$, PC $(3,6.4 \%)$, and infection $(8,17.0 \%)$.

\section{Comparison of the CT Features Between Solitary and Multiple Nodules}

Among the 22 patients with multiple nodular pattern, scattered and clustered nodules were detected in 18 $(81.8 \%)$ and $4(18.2 \%)$ cases, respectively (Figures 1 and 2 ). Table 2 shows the CT features of the detected pulmonary nodules. The diameter of solitary nodules was smaller than the diameter of the largest of the multiple nodules
Table I Patients' Clinical Characteristics

\begin{tabular}{|c|c|c|c|}
\hline Characteristics & $\begin{array}{l}\text { Patients } \\
\text { with } \\
\text { Solitary } \\
\text { Nodules } \\
(n=25)\end{array}$ & $\begin{array}{l}\text { Patients } \\
\text { with } \\
\text { Multiple } \\
\text { Nodules } \\
(n=22)\end{array}$ & $P$-value \\
\hline Gender (Male/Female) & $15 / 10$ & $13 / 9$ & 0.949 \\
\hline Mean age (year) & $50.0 \pm 12.3$ & $43.6 \pm 12.8$ & 0.088 \\
\hline Symptoms (+) & $8(32.0)$ & $13(59.1)$ & 0.062 \\
\hline Fever & I & 4 & \\
\hline Cough & 7 & 7 & \\
\hline Sputum & 4 & 6 & \\
\hline Chest pain & 0 & I & \\
\hline Hemoptysis & 0 & I & \\
\hline $\begin{array}{l}\text { Concomitant basic } \\
\text { diseases }\end{array}$ & $7(28.0)$ & $9(40.9)$ & 0.351 \\
\hline HIV infection & 2 & 5 & \\
\hline Malignant tumors & 3 & 0 & \\
\hline $\begin{array}{l}\text { Chronic kidney } \\
\text { disease }\end{array}$ & 0 & 2 & \\
\hline Kidney transplant & 0 & I & \\
\hline Diabetes & 2 & I & \\
\hline
\end{tabular}

Note: Data are expressed as mean \pm SD or $n(\%)$.

Abbreviation: HIV, human immunodeficiency virus.

$(P=0.000)$. Compared with multiple nodules, a lower number of solitary nodules were located in the subpleural zone $(P=0.031)$ and had a cavity or vacuole $(P=0.049)$ (Figures 1 and 2). A greater number of immunocompromised individuals exhibited a cavity (7/8) compared with cases without a cavity $(9 / 39)(87.5 \%$ vs $23.1 \%, P=0.002)$. Distribution in different lobes, CT patterns, and enhancement of solitary and multiple nodules were all similar in both groups. Compared with single nodular pattern, illdefined border, air bronchogram, and halo sign were slightly more prevalent in the multiple nodular pattern, but their differences were not statistically significant $(P>$ 0.05). Hilar or mediastinal lymph node enlargement, pleural effusion, and pericardial effusion were only observed in few patients with multiple nodular patterns.

\section{CT Features of Solitary Nodules}

Regarding the 25 solitary nodules, $22(88.0 \%)$ and 3 $(12.0 \%)$ cases were solid nodules and mGGNs, respectively. Among the 22 solitary solid nodules, lobulation, spiculation, air bronchogram or vacuole, satellite lesions, and halo signs were detected in $6(27.3 \%), 6(27.3 \%), 10$ $(45.5 \%), 12(54.5 \%)$, and $7(31.8 \%)$ cases, respectively. 


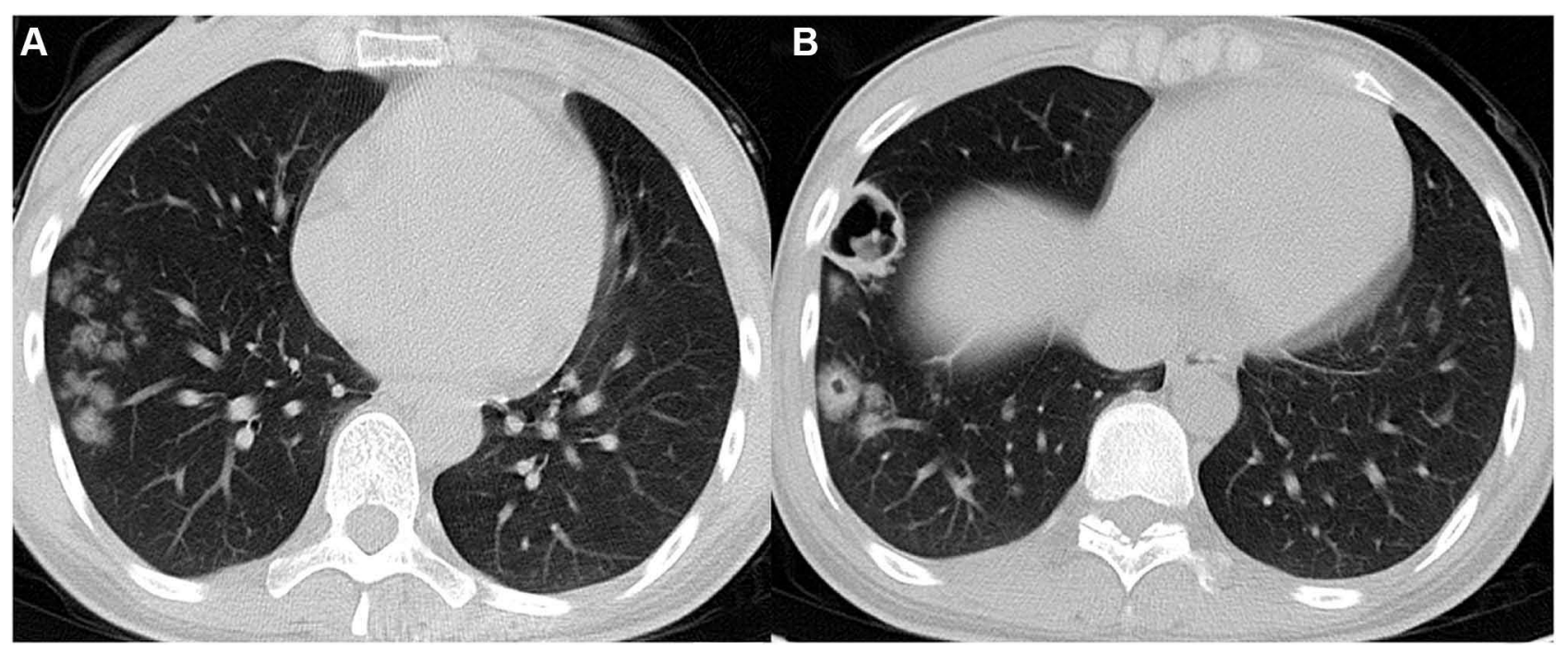

Figure I A 24-year-old woman with HIV infection and pulmonary cryptococcosis. She has headache and fever for ten days, and cryptococcal meningitis is confirmed. (A and B) Axial CT images show multiple clustered nodules with different sizes located in the subpleural zone of right lower lobe. (B) Cavities are detected in the bigger nodules.

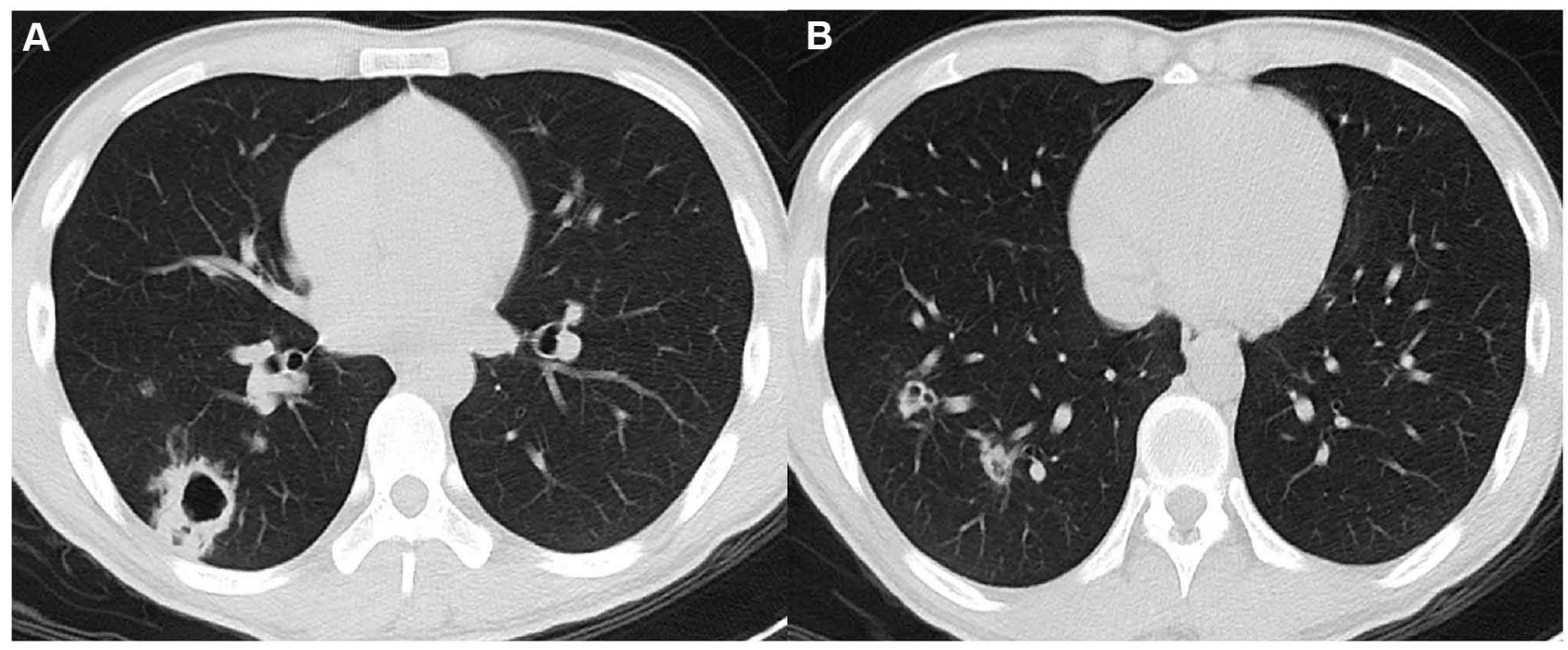

Figure 2 A 24-year-old man with HIV infection and pulmonary cryptococcosis. He has headache and fever for a week, and cryptococcal meningitis is confirmed. (A and B) Axial CT images show multiple scattered nodules with different sizes in the right lower lobe, and cavities are detected in the bigger nodules.

Eighteen (81.8\%) patients demonstrated air bronchogram or vacuole, satellite lesions, and/or a halo sign (Figure 3). In contrast, the mGGNs were all ill-defined, and the internal solid components in two of them were strip-shaped, whereas one demonstrated multiple scattered spots (Figure 4).

\section{Follow-Up results of Solitary Nodules}

Among the 25 patients with solitary nodules, 23 (92.0\%) cases had follow-up CT data due to uncertainty. Comparisons between initial and repeated CT data revealed that nodules in $12(52.1 \%)$ cases had no significant changes in size or density (follow-up time: $3-$ 30 days, median: 13.5 days), 8 (34.8\%) cases increased in size and/or density (follow-up time: 14-210 days, median: 120 days) (Figure 4), and $3(13.0 \%)$ cases decreased in size and/or density (2 accepted anti-inflammatory therapy) (follow-up time: 20-60 days, median: 30 days).

\section{Discussion}

The natural history of cryptococcosis includes two main routes. The first is rare, occurs after exposure to C. neoformans while immunocompromised, leading to rapidly progressive cryptococcosis; the second is common, 
Table 2 CT Characteristics of Solitary and Multiple Nodules

\begin{tabular}{|c|c|c|c|}
\hline Characteristics & Single Nodular Pattern $(n=25)$ & Multiple Nodular Pattern $(n=22)$ & $P$-value \\
\hline Size (range) (mm) & $10.6 \pm 3.5(5-18)$ & $18.0 \pm 6.9(5-29)$ & 0.000 \\
\hline Lobe location & & & 0.136 \\
\hline Right upper lobe & $8(32.0)$ & $7(25.9)$ & \\
\hline Right middle lobe & $2(8.0)$ & I (3.7) & \\
\hline Right lower lobe & $4(16.0)$ & $13(48.2)$ & \\
\hline Left upper lobe & $5(20.0)$ & $2(7.4)$ & \\
\hline Left lower lobe & $6(24.0)$ & $4(14.8)$ & \\
\hline CT patterns & & & 0.164 \\
\hline Solid & $22(88.0)$ & $20(90.9)$ & \\
\hline PGGN & $0(0.0)$ & $\mathrm{I}(4.6)$ & \\
\hline mGGN & $3(12.0)$ & $0(0.0)$ & \\
\hline Solid + GGN & $0(0.0)$ & $\mathrm{I}(4.6)$ & \\
\hline Distribution & & & 0.031 \\
\hline Subpleural zone & $13(52.0)$ & $18(81.8)$ & \\
\hline Non-subpleural zone & $12(48.0)$ & $4(18.2)$ & \\
\hline Boundary & & & 0.358 \\
\hline Well-defined & $15(60.0)$ & $16(72.7)$ & \\
\hline III-defined & $10(40.0)$ & $6(27.3)$ & \\
\hline Cavity and vacuole & $3(12.0)$ & $8(36.4)$ & 0.049 \\
\hline Cavity & I (33.3) & $7(87.5)$ & \\
\hline Vacuole & $2(66.7)$ & $\mathrm{I}(12.5)$ & \\
\hline Air bronchogram & $9(36.0)$ & $6(27.3)$ & 0.522 \\
\hline Halo sign & $7(28.0)$ & $10(45.5)$ & 0.214 \\
\hline Enhancement & & & 0.080 \\
\hline No significant enhancement (<20 HU) & $2(22.2)$ & 0 & \\
\hline Slight enhancement $(20-40 \mathrm{HU})$ & $3(33.3)$ & $9(75.0)$ & \\
\hline Significant enhancement $(>40 \mathrm{HU})$ & $4(44.4)$ & $3(25.0)$ & \\
\hline Hilar or mediastinal lymphadenectasis & 0 & $4(18.2)$ & - \\
\hline Pleural effusion & 0 & $2(9.1)$ & - \\
\hline Pericardial effusion & 0 & I (4.6) & - \\
\hline
\end{tabular}

Note: Data are expressed as mean \pm SD or $n(\%)$.

Abbreviations: GGN, ground glass nodule; pGGN, pure GGN; mGGN, mixed GGN.

has a phase of latency followed by reactivation and dissemination. ${ }^{11} \mathrm{PC}$ results in pulmonary involvement after inhalation of $C$. neoformans spores into the respiratory system. ${ }^{12}$ This condition is prevalent in patients with AIDS and other diseases that compromise immunity. They may undergo primary infection in childhood and a silent phase of latency last for years before reactivation and dissemination. ${ }^{11}$ However, several reports have been published that investigated PC rates in non-AIDS patients and have shown that the incidence of PC in these cases had increased over the last decades. ${ }^{13,14}$ In the present study, approximately $66 \%$ patients had no obvious immune deficiency, which is identical to previous results. ${ }^{4}$ This may be due to the exclusive selection of patients with pulmonary nodules. Pertaining to the detected nodules, especially the solitary ones, they may be stable or increase during follow-up, and thus, they are easy to be misdiagnosed and are worthy of further study.

Clinical manifestations of patients with PC range from no symptom to respiratory failure or acute respiratory distress syndrome depending on host immune responses. ${ }^{15,16}$ However, respiratory symptoms are 


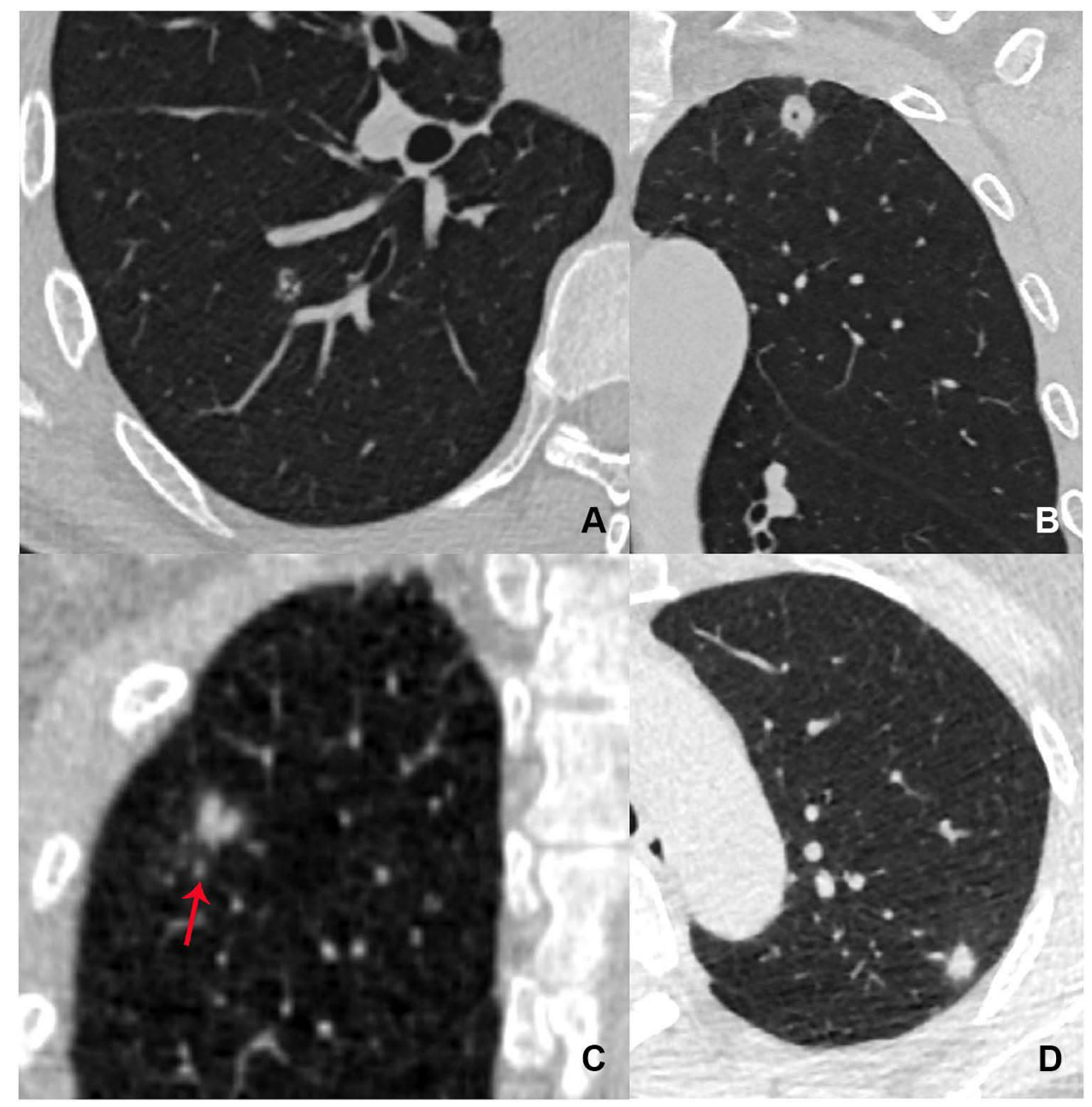

Figure 3 Patients with pulmonary cryptococcosis presented as solitary solid nodules. (A) Axial CT image in a 48-year-old asymptomatic man shows a round and welldefined nodule with small bronchi located in the right upper lobe. (B) Coronal CT image in a 54-year-old asymptomatic man shows a round and well-defined nodule with vacuole located in the left upper lobe. (C) Coronal CT image in a 50-year-old asymptomatic man shows an ill-defined nodule with a satellite lesion (red arrow) located in the right upper lobe. (D) Axial CT image in a 49-year-old asymptomatic woman shows an oval nodule with halo sign located in the left upper lobe.

usually nonspecific, and they are similar to symptoms related to other respiratory diseases. ${ }^{17,18}$ Moreover, a significant portion of asymptomatic cases is detected on chest $\mathrm{CT}$ performed during routine checkups. In this study, more than half of the patients $(55.3 \%)$ presented with no significant respiratory symptoms, especially individuals with solitary nodules. Thus, clinical symptoms could not provide efficient help for the diagnosis of PC nodules. Apart from the respiratory system, cryptococcus has a high affinity for the central nervous system. ${ }^{19}$ In this study, eight patients $(17.0 \%)$ demonstrated cryptococcal meningitis, and the prevalence was higher in patients with multiple nodules. This finding indicates that cryptococcal infection should be considered when differentiating pulmonary nodules in patients with headache.
Chest CT findings of PC can vary, but the most common ones involve solitary or multiple nodules or mass-like opacities, which may reflect histologically pulmonary granuloma or granulomatous pneumonia. ${ }^{20}$ According to a previous study, solitary or multiple pulmonary nodules have been observed in approximately $78 \%$ of non-HIV patients. ${ }^{10}$ Our study revealed a similar result that the majority of patients (85.1\%) had no HIV infection, especially those with solitary nodules $(92.0 \%)$. More specifically, it was shown that the majority of nodules or masses were located in the lower lobes and in the outer zone of the lung field. ${ }^{20}$ In this study, the multiple nodular pattern showed these features, while distribution of those solitary nodules had no significant effect. Additionally, masses or nodules in patients with PC may have central necrosis or liquefaction, thus forming cavities after the release of 


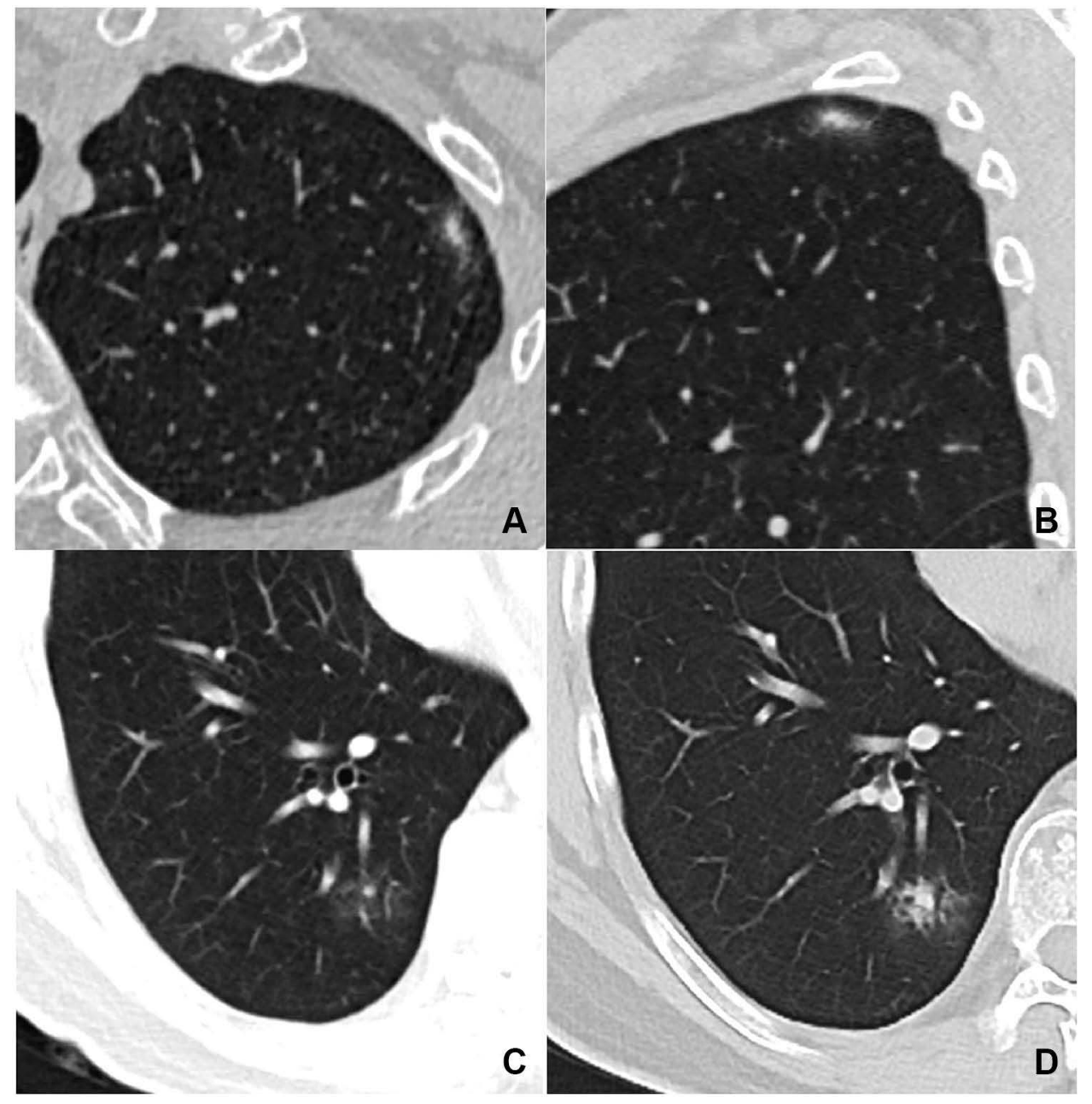

Figure 4 Patients with pulmonary cryptococcosis presented as solitary subsolid nodules. (A and B) Axial and sagittal CT images in a 60-year-old asymptomatic woman show an oval and ill-defined mixed GGN with strip shaped solid component located in the left upper lobe. (C and D) Axial CT images in a 50-year-old asymptomatic man show an ill-defined mixed GGN located in the right lower lobe. (D) The solid component in this nodule significantly increases after one month.

necrotic tissue. ${ }^{17,21}$ The present results indicate that cavities were more commonly detected in multiple nodular pattern and immunocompromised cases, and thus their occurrence may be related to the patients' immune status. Compared with multiple nodules, solitary nodules have less CT features in the above-mentioned aspects. Thus, their diagnosis is significantly more difficult.

Furthermore, the majority of solitary nodules $(88.0 \%)$ in this study were solid, and they had less malignant signs, such as lobulation, spiculation, and pleural indentation. In contrast, air bronchogram or vacuole, satellite lesions, and halo sign were more commonly detected, and $81.8 \%$ had any one of them. Previous studies have also revealed that air-bubble sign, halo sign, and air bronchogram were common CT manifestations of $\mathrm{PC}^{22,23}$ It has been reported that a satellite lesion on chest $\mathrm{CT}$ could be a useful predictor for benign nodules, especially for tuberculomas. ${ }^{24-26}$ In addition, accompanying centrilobular micronodules and cavity or necrosis were more frequently observed in tuberculomas. ${ }^{4}$ However, the present study revealed that a satellite lesion was also frequently observed in solitary nodules caused by PC, in accordance with the results of a previous study. ${ }^{4}$ Thus, for solitary nodules with satellite lesions, PC should be considered as one of the differential diagnosis.

Part solid nodules have been reported to be associated with lung adenocarcinoma, and its malignancy rate is higher than that of pGGNs or solid nodules. ${ }^{27,28}$ A study has reported that larger size, well-defined border, and spiculation favor the diagnosis of malignant lesions, ${ }^{29}$ whereas another research has revealed that lesion multiplicity, large solid portion, and ill-defined border indicate transient lesions. ${ }^{30}$ Additionally, the tumor volume doubling time presented as subsolid nodules is extremely 
long. ${ }^{31}$ In this study, there were three solitary mGGNs, which were not described in detail by previous reports. These three mGGNs were all ill-defined, and the solid components in two of them were strip-shaped and one had multiple scattered spots. During follow-up, the internal solid components in one mGGN increased, whereas another revealed a short-term decrease in its density. Thus, PC should be considered as one of the differential diagnosis for the mGGNs that is characterized by significant short-term changes.

HRCT is useful in the differential diagnosis of pulmonary disease, but it is sometimes difficult to distinguish PC nodules from other benign and malignant nodules. Additionally, fluorine-18 FDG PET/CT and contrast-enhanced CT have limited value in differentiating these nodules because they usually exhibit high FDG uptake and enhancement, thus mimicking malignancy. ${ }^{32}$ Though the clinical and imaging manifestations are nonspecific, the single or multiple nodules should be considered as PC in the following conditions: First, the clinical symptoms do not match with the image findings of pulmonary lesions, especially in cases with multiple nodules but without symptoms. Second, the nodules with air bronchogram, vacuole, satellite lesions, or halo sign cannot be absorbed after general anti-inflammatory or antituberculosis treatment or exhibit short-term increase or decrease in size and/or density. Third, pulmonary nodules, especially the multiple ones, cannot be related or diagnosed with any other definite disease in immunocompromised patients or those presenting with meningitis.

Our study has several limitations. First, this is a retrospective study; thus, a possible selection bias may have compromised the validity of our results. Second, the patient sample size in this study is small. Third, differentiation of solitary PC nodules from primary lung cancer is not studied. Therefore, further investigations to validate our present findings are necessary.

In conclusion, pulmonary nodules were commonly detected in patients with PC. The clinical characteristics of patients with solitary and multiple nodules are similar. On CT images, the solitary nodules are usually smaller, more scattered, but less necrotic than multiple nodules. The majority of solitary nodules were solid, and few of them were mGGNs. PC should be one of the differential diagnosis for the solid nodules with air bronchogram, vacuole, satellite lesions, or halo sign and mixed nodules with ill-defined border, either progressive or stable.

\section{Ethics Statement}

The study was conducted in accordance with the Declaration of Helsinki, and the protocol was approved by the Ethics Committee of the First Affiliated Hospital of Chongqing Medical University (No. 2019-062), which absolved the need for written informed consent because of the retrospective study. All personal identification data were anonymized and de-identified before analysis.

\section{Funding}

This study was supported by the National Natural Science Foundation of China (81601545) and Chongqing Health and Family Planning Commission Foundation (2016MSXM018) of China.

\section{Disclosure}

All authors declare no conflicts of interest for this work.

\section{References}

1. Davies SF, Sarosi GA. Epidemiological and clinical features of pulmonary blastomycosis. Semin Respir Infect. 1997;12:206-218.

2. Woodring JH, Ciporkin G, Lee C, Worm B, Woolley S. Pulmonary cryptococcosis. Semin Roentgenol. 1996;31:67-75. doi:10.1016/ S0037-198X(96)80041-1

3. Nadrous HF, Antonios VS, Terrell CL, Ryu JH. Pulmonary cryptococcosis in nonimmunocompromised patients. Chest. 2003;124:2143-2147. doi:10.1016/S0012-3692(15)31671-8

4. Murayama S, Sakai S, Soeda H, et al. Pulmonary cryptococcosis in immunocompetent patients: HRCT characteristics. Clin Imaging. 2004;28:191-195. doi:10.1016/S0899-7071(03)00145-1

5. Aberg JA, Mundy LM, Powderly WG. Pulmonary cryptococcosis in patients without HIV infection. Chest. 1999;115:734-740. doi:10.1378/chest.115.3.734

6. Chang WC, Tzao C, Hsu HH, et al. Pulmonary cryptococcosis: comparison of clinical and radiographic characteristics in immunocompetent and immunocompromised patients. Chest. 2006;129:333-340. doi:10.1378/chest.129.2.333

7. Haddad N, Cavallaro MC, Lopes MP, et al. Pulmonary cryptococcoma: a rare and challenging diagnosis in immunocompetent patients. Autops Case Rep. 2015;5:35-40. doi:10.4322/acr.2015.004

8. Huang CJ, Yang MC, Ueng SH. Large cryptococcoma mimicking lung cancer in an HIV-negative, type 2 diabetic patient. $J$ Thorac Imaging. 2005;20:115-117. doi:10.1097/01.rti.0000154073.21571.77

9. Lindell RM, Hartman TE, Nadrous HF, Ryu JH. Pulmonary cryptococcosis: CT findings in immunocompetent patients. Radiology. 2005;236:326-331. doi:10.1148/radiol.2361040460

10. Xie X, Xu B, Yu C, et al. Clinical analysis of pulmonary cryptococcosis in non-HIV patients in south China. Int $J$ Clin Exp Med. 2015;8:3114-3119.

11. Alanio A. Dormancy in Cryptococcus neoformans: 60 years of accumulating evidence. J Clin Invest. 2020;130:3353-3360. doi:10.1172/ JCI136223

12. Chang CC, Sorrell TC, Chen SC. Pulmonary Cryptococcosis. Semin Respir Crit Care Med. 2015;36:681-691. doi:10.1055/s-00351562895

13. Song KD, Lee KS, Chung MP, et al. Pulmonary cryptococcosis: imaging findings in 23 non-AIDS patients. Korean $J$ Radiol. 2010;11:407-416. doi:10.3348/kjr.2010.11.4.407 
14. Galanis E, Macdougall L, Kidd S, Morshed M; British Columbia Cryptococcus gattii Working Group. Epidemiology of Cryptococcus gattii, British Columbia, Canada, 1999-2007. Emerg Infect Dis. 2010;16:251-257. doi:10.3201/eid1602.090900

15. Kiertiburanakul S, Wirojtananugoon S, Pracharktam R, Sungkanuparph S. Cryptococcosis in human immunodeficiency virus-negative patients. Int $J$ Infect Dis. 2006;10:72-78. doi:10.1016/j.ijid.2004.12.004

16. Kontoyiannis DP, Peitsch WK, Reddy BT, et al. Cryptococcosis in patients with cancer. Clin Infect Dis. 2001;32:E145-50. doi:10.1086/ 320524

17. Deng H, Zhang J, Li J, Wang D, Pan L, Xue X. Clinical features and radiological characteristics of pulmonary cryptococcosis. J Int Med Res. 2018;46:2687-2695. doi:10.1177/0300060518769541

18. Taniwaki M, Yamasaki M, Ishikawa N, Kawamoto K, Hattori N. Pulmonary cryptococcosis mimicking lung cancer. Lancet Infect Dis. 2019;19:1033. doi:10.1016/S1473-3099(19)30278-6

19. Ribeiro NQ, Santos APN, Emídio ECP, et al. Pioglitazone as an adjuvant of amphotericin B for the treatment of cryptococcosis. Int $J$ Antimicrob Agents. 2019;54:301-308. doi:10.1016/j. ijantimicag.2019.06.020

20. Zhang J, Zhang D, Xue X, Yang L, Chen L, Pan L. Clinical analysis of 16 cases of pulmonary cryptococcosis in patients with normal immune function. Ann Palliat Med. 2020;9:1117-1124. doi:10.21037/apm-20-897

21. Li A, Li Q, Guo C, Zhang Y. Asymptomatic meningitis and lung cavity in a case of cryptococcosis. Am $J$ Case Rep. 2017;18:1140-1144. doi:10.12659/AJCR.905905

22. Xie LX, Chen YS, Liu SY, Shi YX. Pulmonary cryptococcosis: comparison of CT findings in immunocompetent and immunocompromised patients. Acta Radiol. 2015;56:447-453. doi:10.1177/ 0284185114529105

23. Yang R, Yan Y, Wang Y, Liu X, Su X. Plain and contrast-enhanced chest computed tomography scan findings of pulmonary cryptococcosis in immunocompetent patients. Exp Ther Med. 2017;14:4417-4424. doi:10.3892/etm.2017.5096
24. Kim H, Kang SJ, Suh GY, et al. Predictors for benign solitary pulmonary nodule in tuberculosis-endemic area. Korean J Intern Med. 2001;16:236-241. doi:10.3904/kjim.2001.16.4.236

25. Goo JM, Im JG, Do KH, et al. Pulmonary tuberculoma evaluated by means of FDG PET: findings in 10 cases. Radiology. 2000;216:117-121. doi:10.1148/radiology.216.1.r00j119117

26. Totanarungroj $\mathrm{K}$, Chaopotong $\mathrm{S}$, Tongdee T. Distinguishing small primary lung cancer from pulmonary tuberculoma using 64-slices multidetector CT. J Med Assoc Thai. 2012;95:574-582.

27. Lee HJ, Goo JM, Lee CH, Yoo CG, Kim YT, Im JG. Nodular ground-glass opacities on thin-section $\mathrm{CT}$ : size change during follow-up and pathological results. Korean J Radiol. 2007;8:22-31. doi:10.3348/kjr.2007.8.1.22

28. Henschke CI, Yankelevitz DF, Mirtcheva R, et al. CT screening for lung cancer: frequency and significance of part-solid and nonsolid nodules. AJR Am J Roentgenol. 2002;178(5):1053-1057. doi:10.2214/ajr.178.5.1781053

29. Yang W, Sun Y, Fang W, et al. High-resolution computed tomography features distinguishing benign and malignant lesions manifesting as persistent solitary subsolid nodules. Clin Lung Cancer. 2018;19:e75e83. doi:10.1016/j.cllc.2017.05.023

30. Lee SM, Park CM, Goo JM, et al. Transient part-solid nodules detected at screening thin-section CT for lung cancer: comparison with persistent part-solid nodules. Radiology. 2010;255:242-251. doi:10.1148/radiol.09090547

31. Takashima S, Sone S, Li F, Maruyama Y, Hasegawa M, Kadoya M. Indeterminate solitary pulmonary nodules revealed at population-based CT screening of the lung: using first follow-up diagnostic CT to differentiate benign and malignant lesions. AJR Am J Roentgenol. 2003;180:1255-1263. doi:10.2214/ajr.180.5.1801255

32. Wang SY, Chen G, Luo DL, et al. 18F-FDG PET/CT and contrast-enhanced CT findings of pulmonary cryptococcosis. Eur J Radiol. 2017;89:140-148. doi:10.1016/j.ejrad.2017.02.008
Infection and Drug Resistance

\section{Publish your work in this journal}

Infection and Drug Resistance is an international, peer-reviewed openaccess journal that focuses on the optimal treatment of infection (bacterial, fungal and viral) and the development and institution of preventive strategies to minimize the development and spread of resistance. The journal is specifically concerned with the epidemiology of antibiotic resistance and the mechanisms of resistance development and diffusion in both hospitals and the community. The manuscript management system is completely online and includes a very quick and fair peerreview system, which is all easy to use. Visit http://www.dovepress.com/ testimonials.php to read real quotes from published authors. 